\title{
INDECOMPOSABLE POSITIVE MAPS IN MATRIX ALGEBRAS
}

AUTHOR(S):

Tanahashi, Kotaro; Tomiyama, Jun

\section{CITATION:}

Tanahashi, Kotaro ... [et al]. INDECOMPOSABLE POSITIVE MAPS IN MATRIX ALGEBRAS. 数 理解析研究所講究録 1988, 653: 27-41

ISSUE DATE:

1988-04

URL:

http://hdl.handle.net/2433/100484

RIGHT: 
INDECOMPOSABLE POSITIVE MAPS IN MATRIX ALGEBRAS

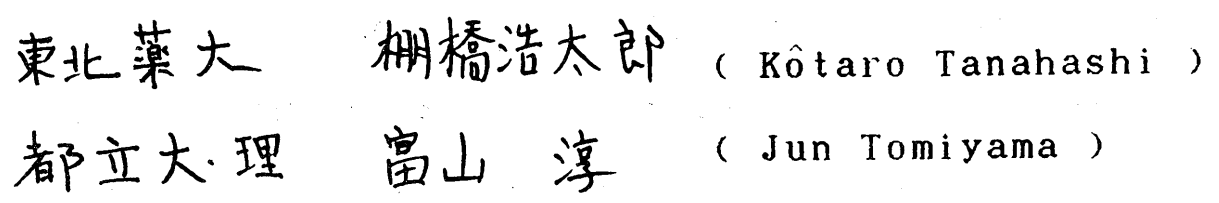

We prove that Choi's map in $M_{3}$ cannot be written as the sum of a 2-positive map and a 2-copositive map. We also provide other examples of positive maps in $M_{n}$ which cannot be written as the sum of an n-positive map and a 2-copositive map.

§1. Introduction.

Let $M_{n}$ be the matrix algebra of order $n$ and $\Phi\left(M_{n}\right)$ be the set of all positive linear maps in $M_{n}$. One of the basic problems about the structure of $P\left(M_{n}\right)$ is whether there exists some small number of simpler convex cones in $\mathcal{P}\left(M_{n}\right)$ with which every positive map can be written as a sum. Two convex cones were proposed as candidates, the cone of completely positive maps and the cone of completely copositive maps. With these cones the program was successful at least for the algebra $M_{2}$ ([9],[14]). That this is not the case for higher dimensional algebras was shown by Choi [3] by an example of an indecomposable positive map in $M_{3}$. Woronowicz [14] also showed the existence of such indecomposable maps.

In this paper we provide examples of positive maps in $M_{n}$ which 
may be considered as proper extensions of Choi's map, and we prove even stronger indecomposability of such maps (Theorem 1 and 4 ). On the other hand, we believe that from the above strategy towards the structure theory of the set $\Phi\left(M_{n}\right)$ what is important is not merely the existence of extremal positive maps but the existence and behavior of those positive maps which are neither 2-positive nor 2-copositive. An example of such an " atomic " positive map in $M_{4}$ has been recently observed by Robertson [8]. We shall show that Choi's map in $M_{3}$ is also atomic. Although this map is known to be extremal by [4], it is not easy to show the property and thus our direct proof of the atomic property (Theorem 5) would be of own interest.

We are deeply indebted to T. Ando for many valuable comments during the preparation of this paper. Thanks are also due to H. Matsuzaki for the estimation of the inequality used below.

§2. Examples of positive maps in $M_{n}$.

Throughout this paper we denote by $\left\{e_{i j}\right\}_{1}^{n}$ the canonical matrix units in the algebra $M_{n}$ for which we always assume that n 23 . The algebra $M_{k}\left(M_{n}\right)$ means the block matrix algebra of order $k$ over $M_{n}$. Let $\tau$ be a linear map in $M_{n}$. The map $\tau$ is said to be k-positive (resp. k-copositive) if the k-multiplicity map $\tau(k)$ ( resp. k-comultiplicity map $\tau^{c}(k)$ ),

$$
\begin{gathered}
\left.\tau(k):\left[a_{i j}\right]_{1}^{k} \in M_{k}\left(M_{n}\right) \underset{i j}{\left(\operatorname{resp} \cdot \tau^{c}(k):\left[a_{i j}\right]_{1}^{k} \in M_{k}\left(M_{n}\right) \longrightarrow\left[\tau\left(a_{i j}\right)\right]_{1}^{k}\right.} \underset{\alpha}{\longrightarrow}\left[\tau\left(a_{j i}\right)\right]_{1}^{k}\right)
\end{gathered}
$$


is positive. If $\tau(k)$ is positive for every $k$, then $\tau$ is said to be completely positive. It is, however, known that in $M_{n}$ positivity of the maps saturates at the order $n$, that is, every n-positive map is completely positive. Completely copositive maps are defined in a similar way and the saturation of copositivity in $M_{n}$ also occurs.

Let $\varepsilon$ be the projection of norm one of $M_{n}$ to the diagonal part and let $s$ be the shift unitary in $M_{n}$ such that

$$
s=\left[\begin{array}{ll}
\delta_{i}+1 &
\end{array}\right]
$$

where indexes are understood to be mod $n$.

A prototype of Choi's map is then written as

$$
\Phi(x)=2 \varepsilon(x)+\varepsilon\left(s x s^{*}\right)-x, \quad x \in M_{3} \text {. }
$$

The map $\Phi$ cannot be written as a sum of a completely positive ( i.e. 3-positive) map and a completely copositive ( i.e. 3-copositive) map. The following map may be regarded as an extension of $\Phi$ to general matrix algebras.

Therem 1. Define the map $\tau_{1}$ in $M_{n}$ by

$$
\tau_{1}(x)=(n-1) \varepsilon(x)+\varepsilon\left(s x s^{*}\right)-x .
$$

Then, $\tau_{1}$ is a positive map.

For the proof we need two lemmas.

Lemma 2. Let a be a positive invertible operator on a Hilbert space and let $\xi_{0}$ be the unit vector associated with a one dimensional projection p. Then a $2 p$ if and only if 
$\left(a^{-1} \xi_{0}, \xi_{0}\right) \leq 1$

The result is rather a standard one, and hence we only mention the equality,

$$
\sup \{(p \xi, \xi) /(a \xi, \xi) \mid\|\xi\|=1\}=\left(a^{-1} \xi_{0}, \xi_{0}\right) .
$$

The next lemma plays a key role in our discussions.

Lemma 3 . Let $\lambda_{1}, \lambda_{2}, \ldots, \lambda_{n}$ be positive numbers. Then

$$
\sum_{i=1}^{n} \frac{\lambda_{i}}{(n-1) \lambda_{i}+\lambda_{i-1}} \leq 1
$$

where we put $\lambda_{0}=\lambda_{n}$.

Proof. Put $a_{i}=\lambda_{i-1} / \lambda_{i}$. Then the inequality changes into the form $\sum_{i=1}^{n} \frac{1}{n-1+a_{i}} \leq 1$ where $\left\{a_{i}\right\}$ are positive numbers with $a_{1} a_{2} \cdots a_{n}=1$. Thus it suffices to show that

$$
\begin{aligned}
& T=\left(n-1+a_{1}\right)\left(n-1+a_{2}\right)\left(n-1+a_{3}\right) \ldots\left(n-1+a_{n}\right) \\
& -1 \quad\left(n-1+a_{2}\right)\left(n-1+a_{3}\right) \ldots\left(n-1+a_{n}\right) \\
& -\left(n-1+a_{1}\right) \quad 1 \quad\left(n-1+a_{3}\right) \ldots\left(n-1+a_{n}\right) \\
& -\left(n-1+a_{1}\right)\left(n-1+a_{2}\right)\left(n-1+a_{3}\right) \ldots \quad 1
\end{aligned}
$$

is non-negative. Let $T_{k}(1 \leq k \leq n-1)$ be the sum of those terms of the form $\lambda\left(i_{1}, i_{2}, \ldots, i_{k}\right) a_{i_{1}} a_{i_{2}} \cdots a_{i_{k}}$ where $\lambda\left(i_{1}, i_{2}, \ldots, i_{k}\right)$ is the coefficient of $a_{i_{1}} a_{i_{2}} \cdots^{\cdots} a_{i_{k}} \cdot$ Notice that here the 
coefficient $\lambda\left(i_{1}, i_{2}, \ldots, i_{k}\right)$ does not depend on the choice of $\left\{a_{i_{1}}, a_{i_{2}}, \ldots, a_{i_{k}}\right\}$. It follows that

$$
\mathrm{T}_{k}=\mu_{k} i_{1}<i_{2}<\cdots<i_{k} a_{i_{1}} a_{i_{2}} \cdots a_{i_{k}}
$$

where

$$
\begin{aligned}
\mu_{k} & =\text { coefficient of } a_{1} a_{2} \cdots a_{k} \\
& =(n-1)^{n-k}-(n-k)(n-1)^{n-k-1} \\
& =(k-1)(n-1)^{n-k-1} \geq 0 .
\end{aligned}
$$

On the other hand, since $a_{1} a_{2} \cdots a_{n}=1$, we have

$$
i_{1}<i_{2}<\cdots<i_{k} a_{i_{1}} a_{i_{2}} \cdots a_{i_{k}} \sum_{n} c_{k} \cdot
$$

Here we note that ${ }_{n} C_{k}$ is the value of the left member when

$$
a_{1}=a_{2}=\cdots=a_{n}=1 \text {. }
$$

Since the other terms are constant with respect to the $a_{i}$ 's, the minimum of $T$ for valuable $a_{i}$ 's is obtained in the above case. Therefore,

$$
T \geq n^{n}-n \cdot n^{n-1}=0
$$

and the proof is completed.

Proof of Theorem 1. It suffices to show that $\tau_{1}(p) \geq 0$ for every one dimensional projection p, that is,

$$
(n-1) \varepsilon(p)+\varepsilon\left(\operatorname{sps}^{*}\right) \geq p \text {. }
$$

Let $\xi_{0}=\left(\alpha_{1}, \alpha_{2}, \ldots, \alpha_{n}\right)$ be the unit vector associated with $p$. Without loss of generality we may assume that $\alpha_{i} \neq 0$ for every i. Then the matrix 


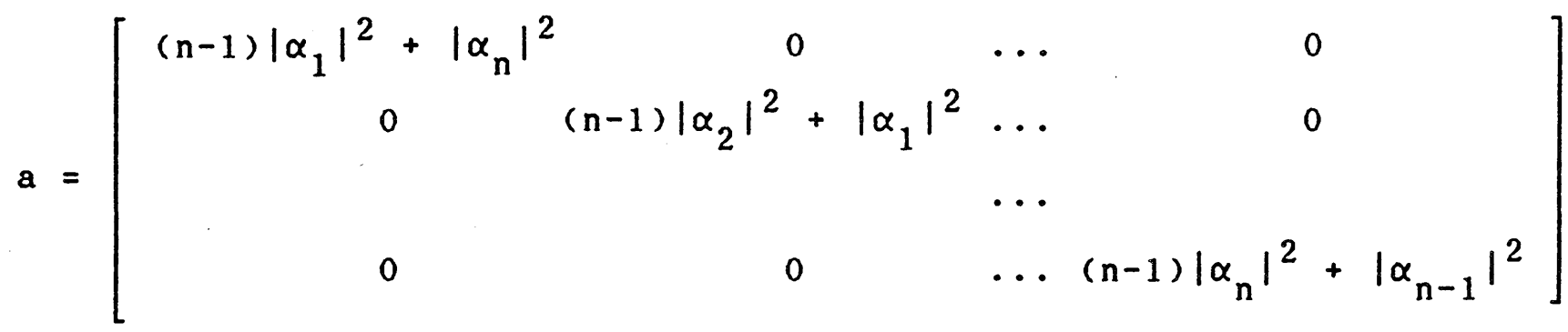
is clearly positive and invertible. It follows by Lemma 2 that the above inequality reduces to the form

$$
\sum_{i=1}^{n} \frac{\left|\alpha_{i}\right|^{2}}{(n-1)\left|\alpha_{i}\right|^{2}+\left|\alpha_{i-1}\right|^{2}}=\left(a^{-1} \xi_{0}, \xi_{0}\right) \leq 1,
$$

but this inequality holds by Lemma 3 . Thus the map $\tau_{1}$ is positive.

There is also an example of a positive map $\tau_{n-2}$ due to Ando [1] which may be considered as another extension of $\Phi$ to $M_{n}$ :

$$
\tau_{n-2}(x)=2 \varepsilon(x)+\sum_{i=1}^{n-2} \varepsilon\left(s^{i} x s^{* i}\right)-x \text {. }
$$

Following an observation due to Y. Nakamura, we suspect that there would be a series of positive maps connecting $\tau_{1}$ and $\tau_{n-2}$, namely,

$$
\tau_{k}(x)=(n-k) \varepsilon(x)+\sum_{i=1}^{k} \varepsilon\left(s^{i} x s^{* i}\right)-x .
$$

However, we have not been able to establish the positivity of these maps, or the indecomposability of $\tau_{n-2}$. Finally, we remark that the map $\tau_{n-1}$ defined as

$$
\tau_{n-1}(x)=\varepsilon(x)+\sum_{i=1}^{n-1} \varepsilon\left(s^{i} x s^{* i}\right)-x
$$

is completely copositive. In fact, as in the case of completely positive maps, completely copositivity of $\tau_{n-1}$ is equivalent to positivity of the matrix

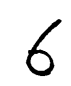




$$
\left[\tau_{n-1}\left(e_{j i}\right)\right]_{1}^{n}=1-\left[e_{j i}\right]_{1}^{n} \text {, }
$$

and as [ $\left.e_{j i}\right]_{1}^{n}$ is a selfadjoint unitary element, this matrix is positive.

§3 Indecomposable positive maps in $M_{n}$.

In this section we study the problem of decomposability of a positive map in $M_{n}$, not only as the sum of an n-positive map and an n-copositive map, but also as a sum of maps in any two categories with higher order of positivity than the original one. With this point of view in mind, we call a positive map in $M_{n}$ an atom if it can not be written as the sum of a 2-positive map and a 2-copositive map. We shall show that there always exists such an atomic positive map in $M_{n}$ if $n \geq 3$. The mutual independence of atomic maps in an appropriate sense and the number of independent atomic maps would be an interesting next problem for the structure of $9\left(M_{n}\right)$, which will be discussed elsewhere.

Theorem 4. Let $k$ be the integer such that $k=\frac{n-1}{2}$ if $n$ is odd and $k=\frac{n}{2}-1$ if $n$ is even. Then the positive map

$$
\tau(x)=(n-1) \varepsilon(x)+\sum_{i=1}^{k} \varepsilon\left(s^{i} x s^{* i}\right)-x
$$

is not decomposable into the sum of an n-positive map and a 2-copositive map.

Proof. Suppose that $\tau=\rho_{1}+\rho_{2}$ for a n-positive map $\rho_{1}$ and a 2-copositive map $\rho_{2}$. Since the matrix $\left[e_{i j}\right]_{1}^{n}$ is positive, the 
matrix

$$
\left[\rho_{1}\left(e_{i j}\right)\right]_{1}^{n}=\left[a_{i j}\right]_{1}^{n}
$$

is positive whereas for the matrix

$$
\left[\rho_{2}\left(e_{i j}\right)\right]_{1}^{n}=\left[b_{i j}\right]_{1}^{n}
$$

every $2 \times 2$ submatrix is the transpose of a positive matrix. Now since $\tau\left(e_{i i}\right)$ is orthogonal to any projection $e_{1 l}$ for $1=i+k+1$, $i+k+2, \ldots, i+n-1$ (where indexes are understood to be mod $n$ ), we have, considering the positivity of $a_{i i}$ and $b_{i i}$,

$$
\mathrm{e}_{11} \mathrm{~b}_{\mathrm{i} i}=\mathrm{b}_{\mathrm{i} i} \mathrm{e}_{11}=0
$$

for $i=1,2, \ldots, n$ and $l=i+k+1, i+k+2, \ldots, i+n-1$.

It follows that

$$
e_{11} b_{j i}=b_{i j} e_{1 l}=0
$$

for every projection $e_{1 l}$ for $j=1,2, \ldots, n$ because

$$
\left[\begin{array}{ll}
b_{i i} & b_{j i} \\
b_{i j} & b_{j j}
\end{array}\right] \geq 0,
$$

and we may assume ( $c f .[6]$ ) that the above matrix is a sum of matrices of the form

$$
\left[\begin{array}{ll}
b_{i}^{*} b_{i} & b_{i}^{*} b_{j} \\
b_{j}^{*} b_{i} & b_{j}^{*} b_{j}
\end{array}\right] \text {. }
$$

Write

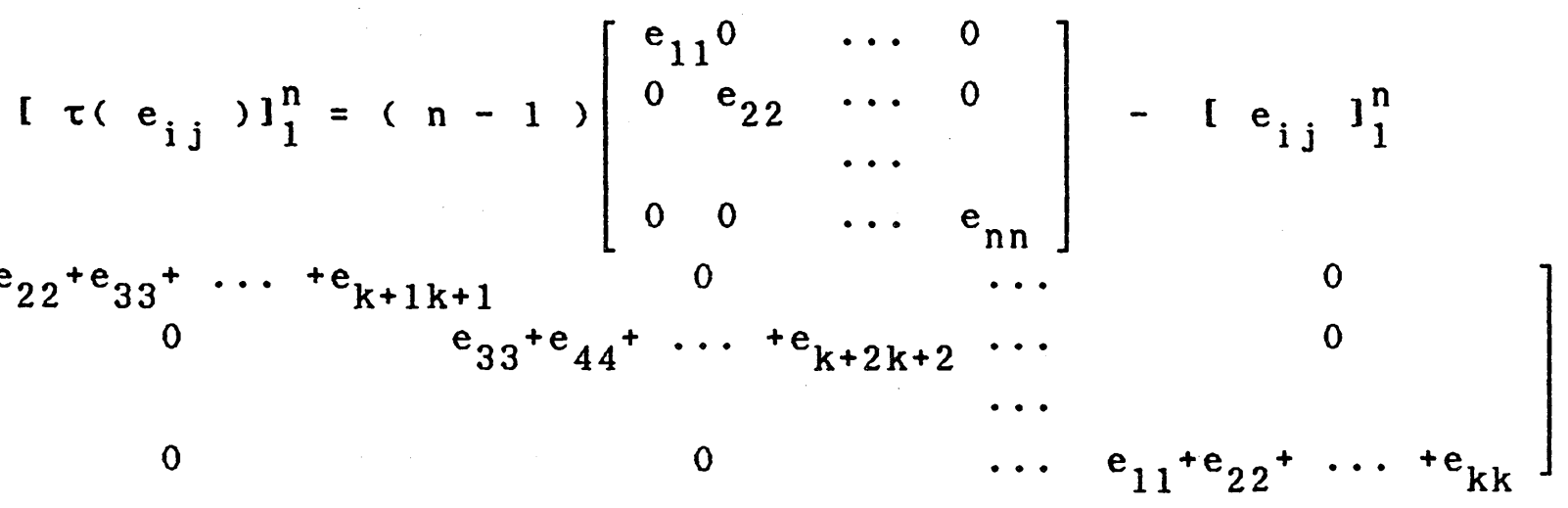

$$
\begin{aligned}
& \text { and note that }\left[e_{i j}\right]_{1}^{n}=n p_{0} \text { for a one dimensional projection } p_{0} \\
& 8
\end{aligned}
$$


which is majorized by the projection $q$ in the first term and is orthogonal to the third projection. Therefore,

$q\left[\tau\left(e_{i j}\right)\right] q=(n-1) q-n p_{0}=(n-1)\left(q-p_{0}\right)-p_{0}$ which is clearly not positive. On the other hand, we assert that the matrix $q\left[b_{i j}\right] q$ is of diagonal form, so that the right member of $q\left[\tau\left(e_{i j}\right)\right] q, q\left(\left[b_{i j}\right]\right) q$ becomes positive, a contradiction. In fact, as $q\left[b_{i j}\right] q=\left[e_{i i} b_{i j} e_{j j}\right], e_{i i} b_{i j} e_{j j}=0$ for $i=$ $1,2, \ldots, n$ and $j=i+k+1, i+k+2, \ldots, i+n-1 \quad($ where indexes are understood to be $\bmod n$ ), we have $e_{i j} b_{i j} e_{j j}=0$ for $i \neq j$ because $b_{i j}=b_{j i}$. This completes the proof.

As an immediate consequence we see that the map $\tau_{1}$ has the same degree of indecomposability. Furthermore, the trivial embedding of Choi's map $\Phi$ into $\Phi\left(M_{n}\right)$ shows that there exists at least one positive map in $M_{n}(n \geq 3)$ which is not written as the sum of a 3-positive map and a 2-copositive map. However, as mentioned above, we can prove a stronger result, namely the existence of an atomic positive map in $M_{n}(n \geq 3)$.

In [14], Woronowicz has proved that there exists an indecomposable positive map $\tau_{w}: M_{2} \longrightarrow M_{4}$ Let $\sigma: M_{4} \longrightarrow M_{2}$ be the norm 1 projection which $\sigma\left(\begin{array}{lll}{[} & a_{i j} & ]_{1}^{4}\end{array}\right)=\left[\begin{array}{ll}a_{11} & a_{12} \\ a_{21} & a_{22}\end{array}\right]$. Then $\tau_{\mathrm{w}} \cdot \sigma: \mathrm{M}_{4} \longrightarrow \mathrm{M}_{4}$ is a positive map which cannot be decomposable into the sum of a 2-positive map and 2-copositive map. Therefore this embedding procedure shows the existence of an atomic map in $M_{n}$ for n $\geq 4$. But unfortunately his proof is not constructive and rather complicated. On the other hand, as mentioned before there is a 
constructive example of an atomic map in $M_{4}$ by [8].

Theorem 5. Choi's map $\Phi$ is not decomposable into the sum of a 2-positive map and a 2-copositive map.

If we make use of the extremal property of $\Phi$ by [4] all what we have to show are Lemma 6 and the fact that $\Phi$ is not 2-copositive. But the following direct proof would be of own interest.

Lemma 6. $\Phi$ is not 2-positive.

Proof. If $\Phi$ were 2-positive, it would satisfy the Schwartz inequal ity,

$$
\Phi(x) * \Phi(x) \leq 2 \Phi\left(x^{*} x\right), \quad x \in M_{3} .
$$

But if we take the matrix

$$
x=\left[\begin{array}{lll}
1 & 0 & 0 \\
1 & 2 & 0 \\
1 & 1 & 1
\end{array}\right]
$$

a straightforward calculation yields that

$$
2 \Phi\left(x^{*} \mathrm{x}\right)-\Phi(\mathrm{x}) * \Phi(\mathrm{x})=\left[\begin{array}{rrr}
2 & -4 & 1 \\
-4 & 6 & 1 \\
1 & 1 & 3
\end{array}\right] \text {. }
$$

and it is easily seen that this matrix is not positive. This shows that the Schwartz inequality for $\Phi$ does not hold.

Proof of Theorem 5. Suppose that $\Phi=\rho_{1}+\rho_{2}$ where $\rho_{1}$ is 2-positive and $\rho_{2}$ is 2-copositive. It is enough to prove that $\rho_{2}=0$, thus contradicting Lemma 6 . We shall use the same notations as in the proof of Theorem 4 for the map $\Phi$, such as 


$$
\left[p_{1}\left(e_{i j}\right)\right]_{1}^{3}=\left[a_{i j}\right]_{1}^{3}, q=\left[\begin{array}{lll}
e_{11} & 0 & 0 \\
0 & e_{22} & 0 \\
0 & 0 & e_{33}
\end{array}\right] \text {, etc. }
$$

Then the same argument as there shows that

$$
e_{33} b_{11}=b_{11} e_{33}=e_{11} b_{22}=b_{22} e_{11}=e_{22} b_{33}=b_{33} e_{22}=0 \text {, }
$$

and

$q\left[\Phi\left(e_{i j}\right)\right]_{1}^{3} q=2 q-\left[e_{i j}\right]_{1}^{3}$

$$
=q\left[a_{i j}\right]_{1}^{3} q+\left[\begin{array}{ccc}
e_{11} b_{11} e_{11} & 0 & 0 \\
0 & e_{22} b_{22} e_{22} & 0 \\
0 & 0 & e_{33} b_{33} e_{33}
\end{array}\right] \text {. }
$$

Multiply by the matrix $2 p^{-}=\left[\begin{array}{lll}\mathrm{e}_{11} & \mathrm{e}_{12} & 0 \\ \mathrm{e}_{21} & \mathrm{e}_{22} & 0 \\ 0 & 0 & 0\end{array}\right]$ from both sides. Then, since the projection $p^{\prime}$ is majorized also by $q$, the first term vanishes, whence

$$
p^{-}\left[a_{i j}\right]_{1}^{3} p^{-}+p^{-}\left[\begin{array}{ccc}
e_{11} b_{11} e_{11} & 0 & 0 \\
0 & e_{22} b_{22} e_{22} & 0 \\
0 & 0 & e_{33} b_{33} e_{33}
\end{array}\right] p^{-}=0
$$

By the 2-positivity of $\rho_{1}$, the first term is positive, and hence it must be zero together with the second term. This implies that

$$
e_{11} b_{11} e_{11}+e_{12} b_{22} e_{21}=0 \text {, }
$$

and, as the $b_{i j}$ 's are positive,

$$
e_{11} b_{11}=b_{11} e_{11}=e_{22} b_{22}=b_{22} e_{22}=0 \text {. }
$$

Similarly, we have that

$$
e_{33} b_{33}=b_{33} e_{33}=0 \text {. }
$$

Therefore,

$$
b_{i i}=e_{i+1} i+1 b_{i i} e_{i+1} i+1=\lambda_{i+1} e_{i+1} i+1 \text {, }
$$

where the index $i$ is understood to be $\bmod 3$, and $0 \leq \lambda_{i} \leq 1$ because

$$
\begin{gathered}
e_{i+1 i+1}=e_{i+1 i+1} a_{i i} e_{i+1 i+1}+b_{i i} \geq b_{i i} \geq 0 . \\
/ /
\end{gathered}
$$


It follows that

$$
\left[a_{i j}\right]_{1}^{3}=\left[\begin{array}{rrr}
e_{11}+\left(1-\lambda_{2}\right) e_{22} & -e_{12} & -e_{13} \\
-e_{21} & e_{22}+\left(1-\lambda_{3}\right) e_{33} & -e_{23} \\
-e_{31} & -e_{32} & e_{33}+\left(1-\lambda_{1}\right) e_{11}
\end{array}\right] \text {, }
$$

and $\rho_{1}$ may be written as

$$
\rho_{1}=2 \varepsilon(x)+\tau^{-}(x)-x
$$

where $\tau^{-}$is a 3-positive map, defined by the matrix

$$
\left[\tau^{\prime}\left(e_{i j}\right)\right]_{1}^{3}=\left[\begin{array}{ccc}
\left(1-\lambda_{2}\right) e_{22} & 0 & 0 \\
0 & \left(1-\lambda_{3}\right) e_{33} & 0 \\
0 & 0 & \left(1-\lambda_{1}\right) e_{11}
\end{array}\right] \text {. }
$$

We now assert that the map $\rho_{1}$ can not be positive unless the $\lambda_{i}$ 's are all zero. In fact, as in the proof of Theorem 1 , the positivity of $\rho_{1}$ is equivalent to the assertion that $2 \varepsilon(p)+\tau^{-}(p)$ $z p$ for every one dimensional projection $p=\left(\bar{\alpha}_{i} \alpha_{j}\right)$, and this is further converted into the inequality $\sum_{i=1}^{3} \frac{1}{2+\mu_{i}{ }^{a}} \leq 1$, by putting $a_{i}=\frac{\left|\alpha_{i-1}\right|^{2}}{\left|\alpha_{i}\right|^{2}}$ and $\mu_{i}=1-\lambda_{i}$. This is equivalent to saying that

$$
\begin{aligned}
\mathrm{T}= & \left(2+\mu_{1} \mathrm{a}_{1}\right)\left(2+\mu_{2} \mathrm{a}_{2}\right)\left(2+\mu_{3} \mathrm{a}_{3}\right) \\
& -\left(2+\mu_{2} \mathrm{a}_{2}\right)\left(2+\mu_{3} \mathrm{a}_{3}\right)-\left(2+\mu_{1} \mathrm{a}_{1}\right)\left(2+\mu_{3} a_{3}\right) \\
& -\left(2+\mu_{1} a_{1}\right)\left(2+\mu_{2} a_{2}\right) \\
= & -4+\mu_{1} \mu_{2} a_{1} a_{2}+\mu_{2} \mu_{3} a_{2} a_{3}+\mu_{3} \mu_{1} a_{3} a_{1}+\mu_{1} \mu_{2} \mu_{3} \geq 0 .
\end{aligned}
$$

However, if one of the $\lambda_{i}$ 's is not zero, the corresponding $\mu_{i}$ is strictly less than 1 and the value of $T$ can not be positive when

$$
a_{1}=a_{2}=a_{3}=1 \text {. }
$$

As this implies that $\rho_{1}$ is not positive, we conclude that all the $\lambda_{i}$ 's are zero. This shows that $\rho_{2}=0$, as desired.

$$
12
$$


3. Concluding remarks.

We have to mention that the situation surrounding the decomposability of positive maps is not so simple. In [12] the second author has analyzed those positive maps sitting on the lines , onnecting the identity map $\sigma$, the transpose map $\theta$, and the completely positive map $\tau(x)=\frac{1}{n} \operatorname{Tr}(x) 1_{n}$ from the point of view of the rank of positivity of those maps. Here, $\sigma$ is clearly completely positive and $\theta$ is known to be plain positive but completely copositive by definition. The analysis of these maps from the point of view of copositivity, however, shows that there exists a completely positive map on the segment between $\theta$ and $\tau$, which is therefore also the sum of a completely positive map and a (nonzero) positive scalar multiple of $\theta$. Similarly, on the ray through the identity map and $\tau$, we can find a segment on which there appears the highest class of positive maps which are both completely positive and completely copositive. Here we emphasize that the ranges of those maps are not commutative, because there are certainly trivial examples of such maps defined by positive functionals on $M_{n}$ (cf.[13]). We also point out the fact (cf.[12]) that a completely positive map as well as a completely copositive map may be decomposable into the sum of positive maps with lower positivity and copositivity.

\section{References}

1. T. Ando, Positivity of certain maps, seminar note 1985 .

$$
13
$$


2. M. D. Choi, Positive linear maps on $C^{*}-a l g e b r a s$, Canad. J. Math., $24(1972), 520-529$.

3 . - Positive semidefinite biquadratic forms, Linear Algebra and Appl., $12(1975), 95-100$.

4. M. D. Choi and T. Y. Lam, Extremal positive semidefinite forms, Math. Ann., 231 (1977), 1-18.

5. - Some assorted inequalities for positive linear maps on $C^{*}$-algebras, J. Operator Theory 4 (1980), 271-285.

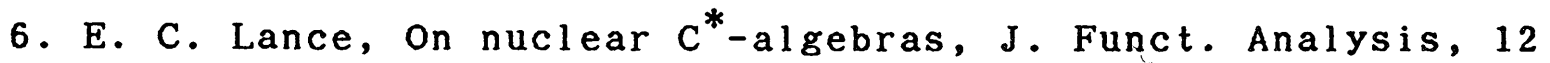
$(1973), 157-176$

7. A. G. Robertson, Schwartz inequalities and decomposition of positive maps on $C^{*}$-algebras, Math. Proc. Camb. Phil. Soc., 94 $(1983), 291-296$

8. Positive projections on $C^{*}-a l g e b r a s$ and an extremal positive map, J. London Math. Soc., 32 (1985), 133-140.

9. E. Stфrmer, Positive linear maps of operator algebras, Acta Math., $110(1963), 233-278$.

10. Decomposable positive maps on $C^{*}$-algebras, Proc. Amer. Math. Soc., $86(1982), 402-404$.

11. J. Tomiyama, on the transpose map of matrix algebras, Proc. Amer. Math. Soc., $88(1983), 635-638$.

12. - On the geometry of positive maps in matrix algebras II, Linear Algebra and Appl., 69 (1985), 169-177.

13. - On the geometry of positive maps in matrix algebras, to appear in the Proceedings of the Conference on Operator Algebras and Mathematical Physics, Iowa, 1985.

14. S. L. Woronowicz, Positive maps of low dimensional matrix 
algebras, Rep. Math. Phys., 10 (1976), 165-183.

K. Tanahashi, Department of Mathematics, Tohoku College of Pharmacy, Komatsushima, Sendai, 983, JAPAN

J. Tomiyama, Department of Mathematics, Tokyo Metropolitan University, Fukasawa, Setagaya-ku, Tokyo, 158, JAPAN 